\title{
Long-term outcomes in half-dose verteporfin photodynamic therapy for chronic central serous retinopathy
}

This article was published in the following Dove Press journal:

Clinical Ophthalmology

\author{
Nadeem Ali Dhirani' \\ Yelin Yang ${ }^{2}$ \\ Sohel Somani ${ }^{2-4}$ \\ 'Wayne State University School \\ of Medicine, Detroit, MI, USA; \\ ${ }^{2}$ Department of Ophthalmology and \\ Vision Sciences, University of Toronto, \\ Toronto, ON, ${ }^{3}$ William Osler Health \\ System, Brampton, ON, ${ }^{4}$ Uptown Eye \\ Specialists, Brampton, ON, Canada
}

Correspondence: Sohel Somani William Osler Health System, Uptown Eye Specialists, 40I-7900 Hurontario Street, Brampton, ON L6Y 0P6, Canada $\mathrm{Tel}+\mathrm{I} 4162920330$

Fax +| 416292033

Email s.somani@utoronto.ca
Objective: To evaluate the short- and long-term outcomes of half-dose verteporfin with photodynamic therapy (PDT) in the treatment of chronic central serous retinopathy (CSR).

Design: Retrospective case series.

Participants: 45 eyes in 39 patients with chronic CSR were included. Diagnosis of chronic CSR was confirmed by fluorescein angiography and persistence of subretinal fluid by optical coherence tomography for a minimum of 3 months duration.

Methods: Each patient underwent treatment with half-dose verteporfin with full-fluence PDT; initial follow-up was defined as a 6-8 week visit following the treatment, and final follow-up ranged from 5 to 70 months.

Results: The average follow-up period for treatment was 19.3 months. Best-corrected visual acuity increased from $\log$ MAR means of 0.52 to $0.42(p<0.05)$. Central retinal thickness and choroidal thickness also significantly decreased at last follow-up $(p<0.05)$. Eight of 45 eyes $(18 \%)$ demonstrated a recurrence of CSR following treatment within the follow-up period. At the final follow-up, 41 out of the 45 eyes (91\%) had complete resolution of subretinal fluid accumulation. Conclusion: Half-dose PDT is an effective treatment option for chronic CSR in a Canadian population, and it is both safe and durable. The positive treatment effect is realized rapidly, with the initial 6-week result highly correlated with the final follow-up result.

Keywords: central serous chorioretinopathy, photodynamic therapy, verteporfin, half-dose verteporfin

\section{Introduction}

Central serous retinopathy (CSR) is characterized by serous detachment of the neurosensory retina and accumulation of subretinal fluid (SRF) in the posterior pole. Although the pathophysiology of the disease is not entirely understood, indocyanine green (ICG) angiography studies have demonstrated choroidal vascular hyperpermeability, suggesting that the primary pathology of CSR occurs at the level of the choroid. ${ }^{1,2}$ Various risk factors have been associated with CSR including, but not limited to, use of corticosteroids, type A personality, pregnancy, and male gender. ${ }^{3}$

Most episodes of CSR resolve spontaneously; however, visual symptoms such as reduced contrast sensitivity or faint scotoma may persist despite the resolution of SRF. $^{3}$ Recurrence has been reported in 30\%-50\% of patients within the first year of the first episode. ${ }^{3}$ Chronic CSR (persistence of SRF $\geq 3$ months) occurs in $5 \%$ of cases and is characterized by widespread retinal pigment epithelium (RPE) changes as well as significant visual morbidity. ${ }^{4}$ 
Various treatment strategies have been described for chronic CSR including laser photocoagulation and medical therapy; however, they do not address the underlying choroidal hyperpermeability. Photodynamic therapy (PDT) has been explored as a treatment option with positive results. It was initially developed for the treatment of choroidal neovascular membranes in age-related macular degeneration. ${ }^{5}$ PDT is thought to act directly on the choroidal vasculature in CSR by reducing choroidal congestion, vascular hyperpermeability, and leakage. ${ }^{6}$ Initial studies using conventional dosage of PDT have shown improved visual outcomes; however, complications including RPE atrophy, choroidal ischemia, and secondary choroidal neovascularization have been described. ${ }^{7,8}$ In an effort to minimize these adverse effects, various modifications to the treatment protocol have been explored including reduced verteporfin dose and fluence settings. ${ }^{69-11}$ However, few studies have compared the short- and long-term outcomes of half-dose verteporfin PDT for treatment of chronic CSR. We aim to describe the short- and long-term outcomes of halfdose verteporfin $\left(3 \mathrm{mg} / \mathrm{m}^{2}\right)$ using standard fluence $\left(50 \mathrm{~J} / \mathrm{cm}^{2}\right)$ PDT in the treatment of chronic CSR.

\section{Methods}

\section{Study design}

This was a retrospective chart review of 45 eyes of 39 patients who had chronic CSR treated at William Osler Health Systems, Brampton, ON, Canada between 2008 and 2015. Inclusion criteria included: 1) diagnosis of chronic CSR confirmed on fluorescein angiography; 2) persistence of SRF on optical coherence tomography (OCT) for a minimum of 3 months duration, with or without concomitant RPE detachment ; 3) hyperpermeability on ICG angiography; 4) treatment with half-dose verteporfin with full-fluence PDT. Exclusion criteria included: previous administration of PDT and the presence of any other confounding macular disease (eg, choroidal neovascular membrane or polypoidal choroidal vasculopathy) that may affect visual acuity. All patients provided written informed consent prior to initiation of any treatment. Ethics approval was obtained from Institutional Research Board Services, ON, Canada (ON IRB registration \#IRB00000776). Any and all private patient information was excluded from the database - and use of this anonymous data was approved by Institutional Review Board services without the need to seek another consent for use of data for research.

After disease confirmation using fluorescein angiography (FA), ICG angiography was used to define the area of abnormal choroidal vascular permeability. Intravenous infusion of half-dose verteporfin $\left(3 \mathrm{mg} / \mathrm{m}^{2}\right)$ for 8 minutes was followed by 83 seconds of full-fluence PDT $\left(50 \mathrm{~J} / \mathrm{m}^{2}\right)$ using the $689 \mathrm{~nm}$ laser. The overall treatment spot size was limited to the smallest circular area that would encompass the choroidal hyperpermeability on ICG angiography, without maximum limit to the spot size selected.

\section{Outcomes}

Baseline metrics collected included best-corrected visual acuity (BCVA); central retinal thickness (CRT), and choroidal thickness (CT). CRT was measured using spectral-domain OCT (Cirrhus OCT; Carl Zeiss Meditec Inc., Dublin, CA, USA). This was obtained manually through measuring the distance between the inner surface of the RPE and the inner surface of the neurosensory retina at the fovea on the horizontal $6.0 \mathrm{~mm}$ scan centered on the fovea. CT was manually assessed by measuring the distance between the outer surface of the RPE and the deepest layer of the choroidal vascular bed visualized on a horizontal subfoveal spectral-domain OCT scan.

Initial follow-up was defined as a 6-8 week visit following the treatment. Final follow-up was defined as the final visit on record. At all of these examinations, the same metrics described in the baseline visit were recorded in addition to evidence of persistent SRF demonstrated on the spectral-domain OCT. Recurrence of CSR was defined as recurrent SRF occurring after complete resolution of SRF during the follow-up period.

\section{Statistical analysis}

Visual acuity values were converted to equivalent logMAR. Primary outcome was the change in BCVA at final follow-up. Secondary measures were changes in CRT, SRF, and CT. Continuous variables were expressed as the mean \pm standard deviation. One-way repeated measures analysis of variance was used to assess changes at different time points. Statistical analysis was performed using Minitab 17 software for Windows, and $p$-values of $<0.05$ was considered to be statistically significant.

\section{Results \\ Baseline demographics}

A total of 45 eyes from 39 patients were included in the study, with bilateral involvement in 16\% (6/39) of patients (Table 1). The mean age was $54.9 \pm 11.7$ years, and male to female ratio was 29:10. The mean duration of active CSR prior to treatment was $7.6 \pm 9.7$ months. The mean period of follow-up was 19.3 \pm 13.0 months (range 5-70 months). Thirty-eight percent (15/39) of patients had presence of active or inactive disease in the contralateral eye.

\section{Primary outcome: BCVA}

Mean logMAR BCVA at baseline, initial ( 68 week) follow-up, and final follow-up was $0.52 \pm 0.38$ (Snellen equivalent $=20 / 66$ ), 
Table I Baseline characteristics of eyes in study

\begin{tabular}{ll}
\hline Variables & $\mathbf{N}=\mathbf{4 5}$ \\
\hline Affected eye (right:left) & $24: 2$ I \\
Mean duration of active CSR (months) & $7.6( \pm 9.7)$ \\
Mean follow-up (mo) & $19.3( \pm 13.0)$ \\
BCVA (logMAR, Snellen) & \\
$\quad$ Baseline & $0.52,20 / 66$ \\
CRT $(\mu \mathrm{m})$ & \\
$\quad$ Baseline & $349( \pm 119)$ \\
CT $(\mu \mathrm{m})$ & \\
$\quad$ Baseline & $311( \pm 43)$ \\
SRF present (n) & \\
$\quad$ Baseline & 45 \\
Initial follow-up & 15 \\
Final & 4 \\
\hline
\end{tabular}

Abbreviations: BCVA, best-corrected visual acuity; CRT, central retinal thickness; CSR, central serous retinopathy; CT, choroidal thickness; SRF, subretinal fluid.

$0.45 \pm 0.42$ (Snellen equivalent $=20 / 56)$, and $0.42 \pm 0.38$ (Snellen equivalent $=20 / 52$ ), respectively (Figure 1 ). There was a significant improvement in BCVA over the course of follow-up ( $p<0.05$ ), with the majority of improvement seen in the initial 6-8 weeks. Six eyes had loss of BCVA by two or more Snellen lines at the last follow-up visit, of which three eyes had recurrence of SRF.

\section{Secondary outcome: anatomical}

Complete resolution of SRF occurred in 67\% (30/45) of eyes at 6-8 week follow-up, and in $91 \%(41 / 45)$ of eyes at the last follow-up (Figure 2).

Baseline mean CRT values were 349 $120 \mu \mathrm{m}$ (Figure 3). There was a statistically significant reduction in CRT of $28 \%$ at initial follow-up $(251 \pm 106 \mu \mathrm{m})$ and $34 \%$ at final follow-up $(229 \pm 61 \mu \mathrm{m})$, respectively $(p<0.05)$.

Baseline mean CT values were $311 \pm 43 \mu \mathrm{m}$ (Figure 4). There was a statistically significant reduction in CT of $22 \%$ at initial follow-up $(242 \pm 42 \mu \mathrm{m})$ and $26 \%$ at final follow-up $(229 \pm 38 \mu \mathrm{m})$, respectively, $(p<0.05)$.

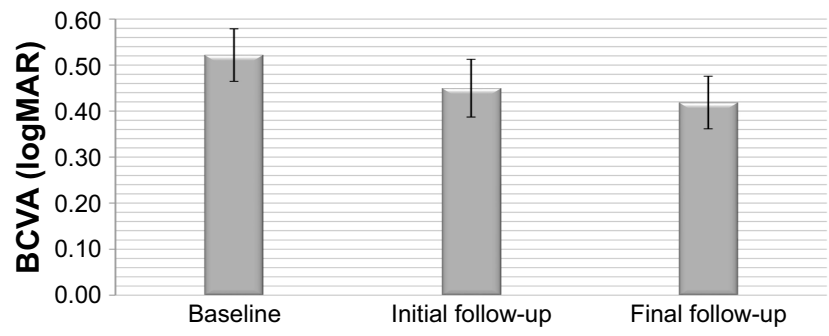

Figure I Mean BCVA at baseline and both follow-ups, showing significant improvement over time.

Note: Error bars represent standard errors of the mean.

Abbreviation: BCVA, best-corrected visual acuity.

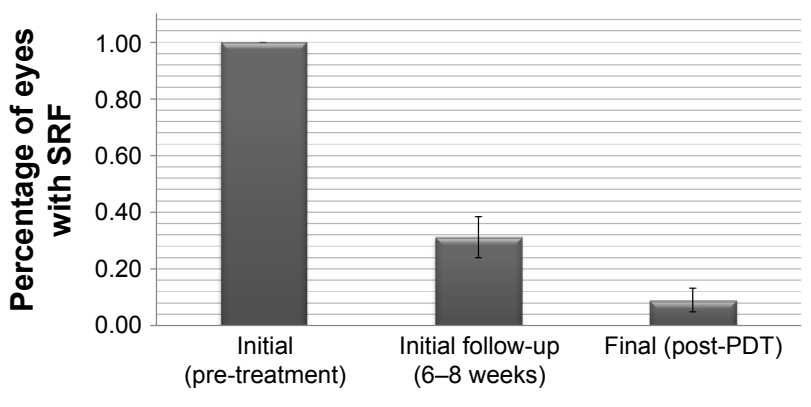

Figure 2 Resolution of subretinal fluid with treatment over time. Abbreviations: PDT, photodynamic therapy; SRF, subretinal fluid.

\section{Correlations}

A comparison of initial follow-up metrics and final follow-up metrics was conducted. There were statistically significant correlations found between the initial follow-up and final follow-up values for BCVA $(R=0.61)$, CRT $(R=0.65)$, and CT $(R=0.79)$, respectively $(p<0.01)$.

\section{Recurrence}

Eight of 45 eyes had recurrence of CSR during the follow-up period as verified through spectral-domain OCT imaging. Three of these eyes had bilateral disease. Treatment for these eyes included observation $(n=5)$, thermal laser $(n=2)$, and/or repeat half-dose PDT $(n=1)$. Four of eight recurrences resolved within the follow-up period.

\section{Discussion}

Although CSR is usually self-limiting, a subset of patients can develop significant visual impairment. It is important to develop a treatment protocol that best addresses the underlying pathophysiology with minimal complications. We report that PDT with half-dose verteporfin is an effective and safe treatment for chronic CSR patients in the long term.

PDT was first described for treatment of CSR using standard dosing protocols $\left(6.0 \mathrm{mg} / \mathrm{m}^{2}, 50 \mathrm{~J} / \mathrm{cm}^{2}\right)$ based on

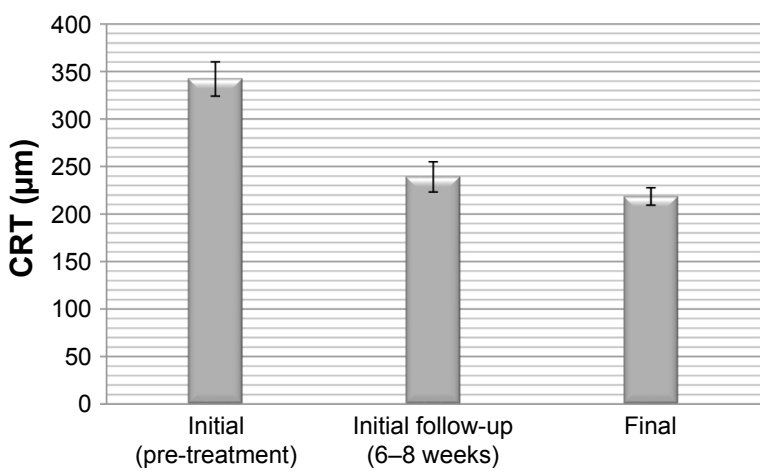

Figure 3 Mean central retinal thickness at baseline and both follow-ups, showing significant reduction with treatment.

Abbreviation: CRT, central retinal thickness. 


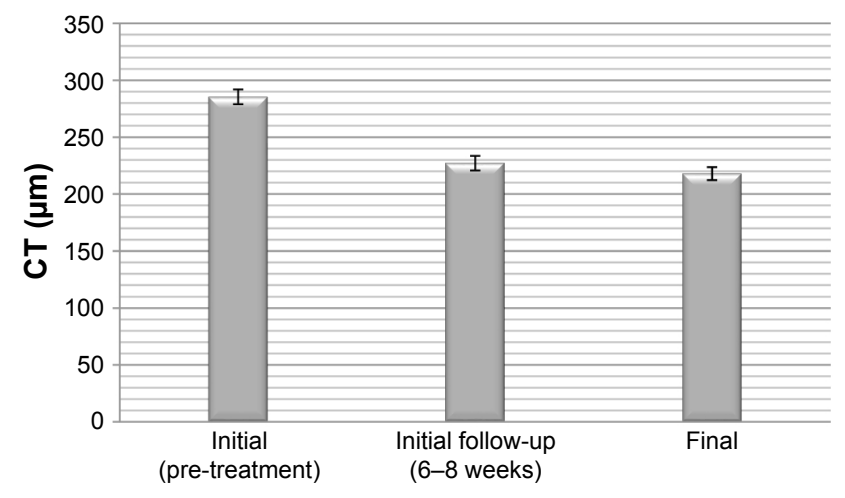

Figure 4 Mean choroidal thickness at baseline and both follow-ups, showing significant reduction with treatment.

Abbreviation: CT, choroidal thickness.

VIP/TAP trials. ${ }^{7,12}$ Despite the demonstrated benefits using full-dose PDT in the CSR population, adverse effects, albeit rare, have been reported in the general PDT literature including transient vision loss, transient multifocal electroretinogram changes, RPE atrophy, RPE tear, secondary choroidal neovascular membrane, choroidal ischemia, and choroidal infarction. ${ }^{11}$ In an effort to minimize these complications, various modified PDT protocols have been explored involving reduced verteporfin dosage, laser fluence, or a combination of both. Studies investigating half-fluence, ${ }^{9,10}$ half-dose, ${ }^{6,11}$ half-fluence and micropulse, ${ }^{13} 1 / 3$ dose, ${ }^{14}$ and minimal-fluence ${ }^{15}$ protocols have all demonstrated some degree of treatment effect. Half-dose verteporfin and half-fluence treatments are the two most described modified protocols. Previous studies that have compared the two treatments demonstrate similar visual outcomes, perhaps with a faster resolution in the half-dose group. ${ }^{16-18}$

Our study further emphasizes the benefit of half-dose PDT in the treatment of chronic CSR at improving both functional and anatomic outcomes. We found a significant improvement in BCVA after treatment, which was noted in the early follow-up visit and sustained until the final follow-up visit. This is consistent with other studies of halfdose PDT, all of which demonstrated significant improvement in visual acuity over the follow-up period, with most patients manifesting improvement early on. ${ }^{11}$ Compared to these previous studies, our study cohort had lower baseline visual acuity scores (logMAR 0.52), and lower final VA scores (logMAR 0.42), likely an indication of underlying RPE damage from chronic disease.

Ninety-one percent of patients in our study had complete resolution of SRF. This was consistent with other studies which demonstrated a range of SRF resolution of $92 \%-93 \%$ with half-dose verteporfin, ${ }^{11,19}$ and $86 \%-91 \%$ in the halffluence protocol. ${ }^{9,10}$ The resolution of SRF in the acute CSR population using PDT has been reported to be slightly higher likely on the basis of less underlying disease severity. ${ }^{6}$ Regardless of SRF duration, these results suggest that half-dose PDT is a viable treatment option for both acute and chronic CSR. Nonetheless, it has been suggested that earlier intervention may be preferred due to better vision outcomes. ${ }^{11}$

The elimination of SRF, and the corresponding decrease in CRT, would imply a favorable response to PDT. The final average CRT of $229 \mu \mathrm{m}$ is consistent with other reported studies in the half-dose ${ }^{6,10}$ and half-fluence ${ }^{9,10}$ literature. A favorable and robust CRT measurement after a mean of 19 months follow-up posttreatment in our study suggests not only durability of results but, more importantly, lack of significant induced RPE atrophy. While RPE atrophy is a known complication of CSR, to date there has been no reported increase in RPE atrophy with half-dose PDT treatment compared to control patients. ${ }^{11}$

A reduction in $\mathrm{CT}$ has been a demonstrated effect of PDT in previous protocols. ${ }^{18,20}$ Our paper further reaffirms the statistically significant effect of half-dose PDT on CT in patients with chronic CSR $(311 \mu \mathrm{m}$ to $229 \mu \mathrm{m})$. The majority of the CT reduction occurred within the initial 6-8 weeks (311 $\mu \mathrm{m}$ to $242 \mu \mathrm{m})$, with a further small reduction noted at the last follow-up $(229 \mu \mathrm{m})$.

Recurrence of disease is a known but uncommon occurrence post PDT. Eighteen percent of patients had recurrence of CSR within the follow-up period. This was within a similar range to other studies in half-dose verteporfin $(18 \%-20 \%))^{10,11}$ The majority of these recurrences occurred in patients with bilateral disease, who likely had more aggressive baseline CSR disease. A previous study reported that patients with recurrent or incomplete SRF absorption had a greater number of previous CSR treatment attempts, suggesting a more chronic refractory disease state. ${ }^{20}$

Significant correlations were identified in all outcome metrics comparing initial follow-up and final follow-up visits. This suggests an early and sustained response to treatment across these functional and anatomic outcomes. The rapid effect of PDT is in line with other studies. ${ }^{6,11}$ Interestingly, a previous study demonstrated that the rate of decline in subfoveal CT at 1-month post PDT is most predictive of recurrence or treatment failure. ${ }^{20}$

There are several limitations within this study. As a retrospective review, the lack of a control group may limit generalizations and limit definitive comparisons of half-dose PDT with other treatment modifications. Nonetheless, this does not detract from the observations of this study cohort demonstrating favorable outcomes after half-dose PDT, with safe and durable effects noted as early as 6 weeks posttreatment. Second, some variations did exist in the exact initial follow-up 
interval among the study cohort, with measurements ranging from 6 to 8 weeks following initial treatment. While it is unlikely these differences provided much skew to our findings, further studies may benefit from having a more standardized period of time for the initial follow-up. Third, the majority of eyes did not undergo subsequent assessment with ICG angiography or intravenous fluorescein angiography to confirm absence or reversal of increased choroidal permeability or leakage. However, given the invasiveness of this diagnostic test, it was deemed impractical to carry this out on patients once clinical benefit was demonstrated on OCT.

\section{Conclusion}

In conclusion, we report that half-dose PDT may be an effective treatment option for chronic CSR that is both safe and durable. The positive treatment effect is realized rapidly, with the initial 6-week result highly correlated with the final follow-up result. Further clinical trials are warranted for evaluating half-dose PDT in this population and potentially comparing to other treatment protocols including full-dose verteporfin and half-fluence PDT.

\section{Acknowledgment}

Presented at the Canadian Ophthalmology Society Annual Meeting, June 20th 2016, Ottawa, ON, Canada.

\section{Disclosure}

The authors report no conflicts of interest in this work.

\section{References}

1. Piccolino FC, Borgia L, Zinicola E, Zingirian M. Indocyanine green angiographic findings in central serous chorioretinopathy. Eye (Lond). 1995;9 (Pt 3):324-332.

2. Nicholson B, Noble J, Forooghian F, Meyerle C. Central serous chorioretinopathy: update on pathophysiology and treatment. Surv Ophthalmol. 2013;58(2):103-126.

3. Liew G, Quin G, Gillies M, Fraser-Bell S. Central serous chorioretinopathy: a review of epidemiology and pathophysiology. Clin Exp Ophthalmol. 2013;41(2):201-214.

4. Wang M, Munch IC, Hasler PW, Prunte C, Larsen M. Central serous chorioretinopathy. Acta Ophthalmol. 2008;86(2):126-145.

5. Photodynamic therapy of subfoveal choroidal neovascularization in age-related macular degeneration with verteporfin: One-year results of 2 randomized clinical trials - TAP report. treatment of age-related macular degeneration with photodynamic therapy (TAP) study group. Arch Ophthalmol. 1999;117(10):1329-1345.

Clinical Ophthalmology

\section{Publish your work in this journal}

Clinical Ophthalmology is an international, peer-reviewed journal covering all subspecialties within ophthalmology. Key topics include: Optometry; Visual science; Pharmacology and drug therapy in eye diseases; Basic Sciences; Primary and Secondary eye care; Patient Safety and Quality of Care Improvements. This journal is indexed on
6. Chan WM, Lai TY, Lai RY, Liu DT, Lam DS. Half-dose verteporfin photodynamic therapy for acute central serous chorioretinopathy: One-year results of a randomized controlled trial. Ophthalmology. 2008;115(10):1756-1765.

7. Cardillo Piccolino F, Eandi CM, Ventre L, Rigault de la Longrais RC, Grignolo FM. Photodynamic therapy for chronic central serous chorioretinopathy. Retina. 2003;23(6):752-763.

8. Chan WM, Lam DS, Lai TY, Tam BS, Liu DT, Chan CK. Choroidal vascular remodelling in central serous chorioretinopathy after indocyanine green guided photodynamic therapy with verteporfin: a novel treatment at the primary disease level. Br J Ophthalmol. 2003;87(12): 1453-1458.

9. Reibaldi M, Cardascia N, Longo A, et al. Standard-fluence versus low-fluence photodynamic therapy in chronic central serous chorioretinopathy: A nonrandomized clinical trial. Am J Ophthalmol. 2010; 149(2):307-315.e2.

10. Nicolo M, Eandi CM, Alovisi C, et al. Half-fluence versus half-dose photodynamic therapy in chronic central serous chorioretinopathy. Am J Ophthalmol. 2014;157(5):1033-1037.

11. Lai TY, Wong RL, Chan WM. Long-term outcome of half-dose verteporfin photodynamic therapy for the treatment of central serous chorioretinopathy (an american ophthalmological society thesis). Trans Am Ophthalmol Soc. 2015;113:T8.

12. Yannuzzi LA, Slakter JS, Gross NE, et al. Indocyanine green angiography-guided photodynamic therapy for treatment of chronic central serous chorioretinopathy: A pilot study. Retina. 2003;23(3): 288-298.

13. Breukink MB, Mohr JK, Ossewaarde-van Norel A, et al. Half-dose photodynamic therapy followed by diode micropulse laser therapy as treatment for chronic central serous chorioretinopathy: evaluation of a prospective treatment protocol. Acta Ophthalmol. 2016;94(2): 187-197.

14. Uetani R, Ito Y, Oiwa K, Ishikawa K, Terasaki H. Half-dose vs onethird-dose photodynamic therapy for chronic central serous chorioretinopathy. Eye (Lond). 2012;26(5):640-649.

15. Butler AL, Fung AT, Merkur AB, Albiani DA, Forooghian F. Very minimal fluence photodynamic therapy for chronic central serous chorioretinopathy. Can J Ophthalmol. 2012;47(1):42-44.

16. Kim YK, Ryoo NK, Woo SJ, Park KH. Comparison of visual and anatomical outcomes of half-fluence and half-dose photodynamic therapy in eyes with chronic central serous chorioretinopathy. Graefes Arch Clin Exp Ophthalmol. 2015;253(12):2063-2073.

17. Cheng $\mathrm{CK}$, Chang $\mathrm{CK}$, Peng $\mathrm{CH}$. Comparison of photodynamic therapy using half-dose of verteporfin or half-fluence of laser light for the treatment of chronic central serous chorioretinopathy. Retina. 2017; 37(2):325-333.

18. Alkin Z, Perente I, Ozkaya A, et al. Comparison of efficacy between low-fluence and half-dose verteporfin photodynamic therapy for chronic central serous chorioretinopathy. Clin Ophthalmol. 2014;8: 685-690.

19. Alkin Z, Yilmaz I, Ozkaya A, Yazici AT. Steroid-induced central serous chorioretinopathy in a patient with non-arteritic anterior ischemic optic neuropathy. Saudi J Ophthalmol. 2015;29(3):232-234.

20. Kim YK, Ryoo NK, Woo SJ, Park KH. Choroidal thickness changes after photodynamic therapy and recurrence of chronic central serous chorioretinopathy. Am J Ophthalmol. 2015;160(1):72-84.e1.

\section{Dovepress}

PubMed Central and CAS, and is the official journal of The Society of Clinical Ophthalmology (SCO). The manuscript management system is completely online and includes a very quick and fair peer-review system, which is all easy to use. Visit http://www.dovepress.com/ testimonials.php to read real quotes from published authors. 